\title{
Agent-Based Modeling and Simulation of Connected Corridors- Merits Evaluation and Future Steps
}

\author{
Montasir Abbas, Ph.D., P.E. ${ }^{1}$ and Sahar Ghanipoor Machiani, Ph.D. ${ }^{2}$ \\ ${ }^{1}$ Associate Professor, Department of Civil and Environmental Engineering, \\ Virginia Tech \\ ${ }^{2}$ Research Associate, Virginia Tech Transportation Institute \\ 'abbas@vt.edu, ${ }^{2}$ sahargh@vt.edu
}

\begin{abstract}
Agent based modeling focuses on simulating the simultaneous operations and interactions of individual entities as opposed to system level simulation. Simple behavioral rules are specified for agents in interaction with the environment, resulting in the generation of complex system behavior. Growing the popularity of this approach in recent years, it has been used in variety of scientific applications. Agents can be people, companies, projects, vehicles, products, etc., making it possible to apply the concept to different grounds of research. This paper presents a background of the agent based modeling, focusing on MATSim (a large-scale agent-based transport simulation tool). An application of MATSim to a large-scale scenario of San Francisco Bay Area is discussed and presented under an integrated connected corridor framework. The simulated network, consisted of 31,608 nodes and 73,711 links, modelled about 2.1 million agents in 1454 TAZs. The result of the simulation is illustrated and future steps are provided.
\end{abstract}

Keywords: Larg-scale agent based modelling; MATSim; San Fransisco Bay Area model

\section{Introduction}

This paper summarizes an effort to investigate the merits of using agent-based modeling and simulation techniques in a connected corridor. Since a connected corridor attempts to address many aspects of network modeling, traveler behavior (mode and route choice), as well as the application and impact of different control strategies, it made sense to look into existing tools that could be used for connected corridors, and evaluate their merits and cost. The following sections provide a description of the objectives and scope of work in this analysis. Next, a literature review of agent-based modeling and simulation techniques used in related efforts is presented, coupled with a background description of the agent based modeling concepts and potential benefits. A description of the software used in this evaluation (MATSIM) is then presented, including the concepts, capabilities, and data needs of the model. The base case model of the Bay area is then described, including the description of the data requirements and needs. The results of the runs are included, with descriptions and explanations of the outcomes. Finally, a conclusion of the paper is provided and possible future steps are identified in the last section of the paper.

\section{Objectives and Scope of Work}

The motivation of this effort was to investigate the merits of using agent-based modeling techniques for modeling and simulation of a connected corridor demand and supply strategies. The objectives of the effort were as follows:

Evaluate the merits of using agent based modeling and simulation (ABMS) concepts in a connected corridor, 
Identify potential advantages of agent-based modeling techniques and determine possible future steps in this area.

\section{Background}

Agent-based modeling and simulation (ABMS) is a relatively new paradigm that describes a system from the perspective of its constituent units [1]. Unlike to "top-down" modeling approach, ABMS is bottom-up approach, where many agents (or decision makers) exhibit different levels of dispersed control, organizational levels. ABMS can be characterized by the ability of agents to adapt and use internal models to anticipate the future [2]. Agents use rules or equations to represent individual behaviors. An ABMS system starts with a set of rules and uses those rules to model the behavior of the overall system.

ABMS is best applied when interactions between agents are complex, nonlinear, discontinuous or discrete. Also, when space is crucial and agents' positions are not fixed, when each individual is different, and when topology of interactions is heterogeneous and complex with different agents exhibiting different behaviors, including learning and adaptation [1]. Agent based modeling has advantages based on the belief that agents are able to interact. Agent behaviors are varied, and the ease of experimentation and computing and ease of bring into implement changes in the system. Two circulars published by the Transportation Research Board (TRB) Artificial Intelligence and Advanced Computing Application committee $[3,4]$ provide useful introductory overview of the concept, structure and application of agent-based modeling in transportation as illustrated in (Figure 1).

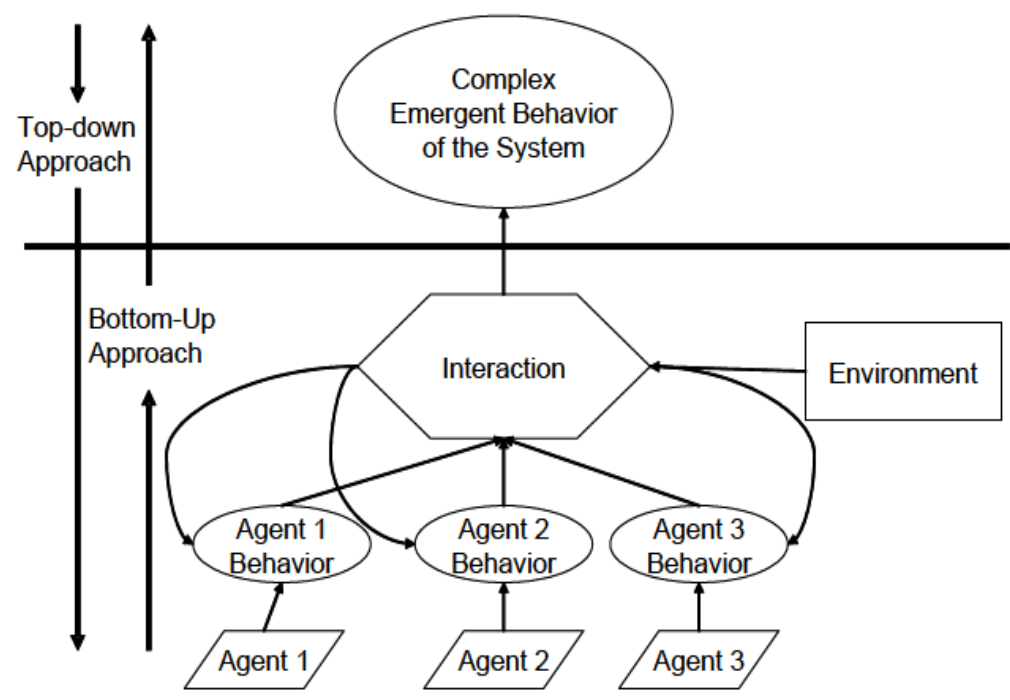

Figure 1. "Bottom-up" Modeling of System [3]

The agent-based modeling method is widely used in various areas of transportation including simulation of vehicles or pedestrian flow, route choice modeling, lane changing and car-following models, and traffic simulation. The first TRB circular refers to two known simulation models as agent-based models (TRANSIMS and MATSIM)[3].

For agent-based modeling, types, attributes, allowable values of attributes, and initial values are defined first. Once the agents are defined, the model needs to specify the interaction rules for the agents so the agents will interact with one another and with their environment. An agent-based model can then be programmed in the developer's programming language or software package of choice. 


\subsection{Why agent-based Modeling and Simulation}

An ABMS paradigm is one where everything could be thought of and abstracted as a representative agent. For example, every person in the network could be modeled as an agent. Network elements could be modeled (or thought of) as agents. Decision makers or meta-controllers could be treated and modeled as agents, etc. ABMS scheme would therefore provide an environment where interaction of disparate entities could be modeled. Traditional microscopic simulation could be thought of as a simplified ABMS system, where agents (vehicles) are only interacting with lead vehicles, and without changes or evolutions in their driving rules. Analysts with computer programming experience could think of agents as objects, in an object-oriented paradigm. Only that, an agent is an object with "intelligence," with rules that could be determined off-line, or online with some learning rules to change the initial rules.

With this in mind, one can see why an ABMS approach could be useful to model a connected corridor environment, with different entities (travelers, pedestrians, drivers on the demand side, and network links, controllers, and strategies on the supply side). In addition, travelers in real life learn and adapt to changes in network - a trend that need to be taken into account in analysis. The evolution of behavior could and should also be modeled on the supply side, when control intelligence is involved.

\subsection{Vision and Methodology}

Figure 2 shows the overall vision of a connected corridor from the writer's perspective. An intelligent decision support system or meta-controller is shown in the figure to consist of a demand management and control subsystems. The demand management subsystem should be able to sense the current state of traffic and predict future states. A control subsystem should be able to devise intelligent strategies for the future states. The metacontroller is shown to interface with either a simulated network or a real network (with a software interface switch) to either study different scenarios or interfere with the real world with verified scenarios. A real world network agent-based model is shown in the heart of the figure. This abstraction of the network allows experimentation with different strategies/models without interfering with the real world. An agent-based modeling and simulation platform (e.g., MATSIM/TRANSIMs) could serve the purpose of abstraction by allowing the interaction of different agents. An ABMS platform can be used to simulate a whole network by simulating a population (every person in a region is represented by an agent), generating daily activities for each agent, determining their best routes and mode choices, and loading them into the network. The supply side can be represented by simulating controllers, ramp meters, and transit schedules. The simulator creates an environment where all agents can interact and where the system behavior can be summarized and studied. The left side of the figure shows where different research efforts could be interfaced with this framework to improve different modeling/simulation component fidelities. 


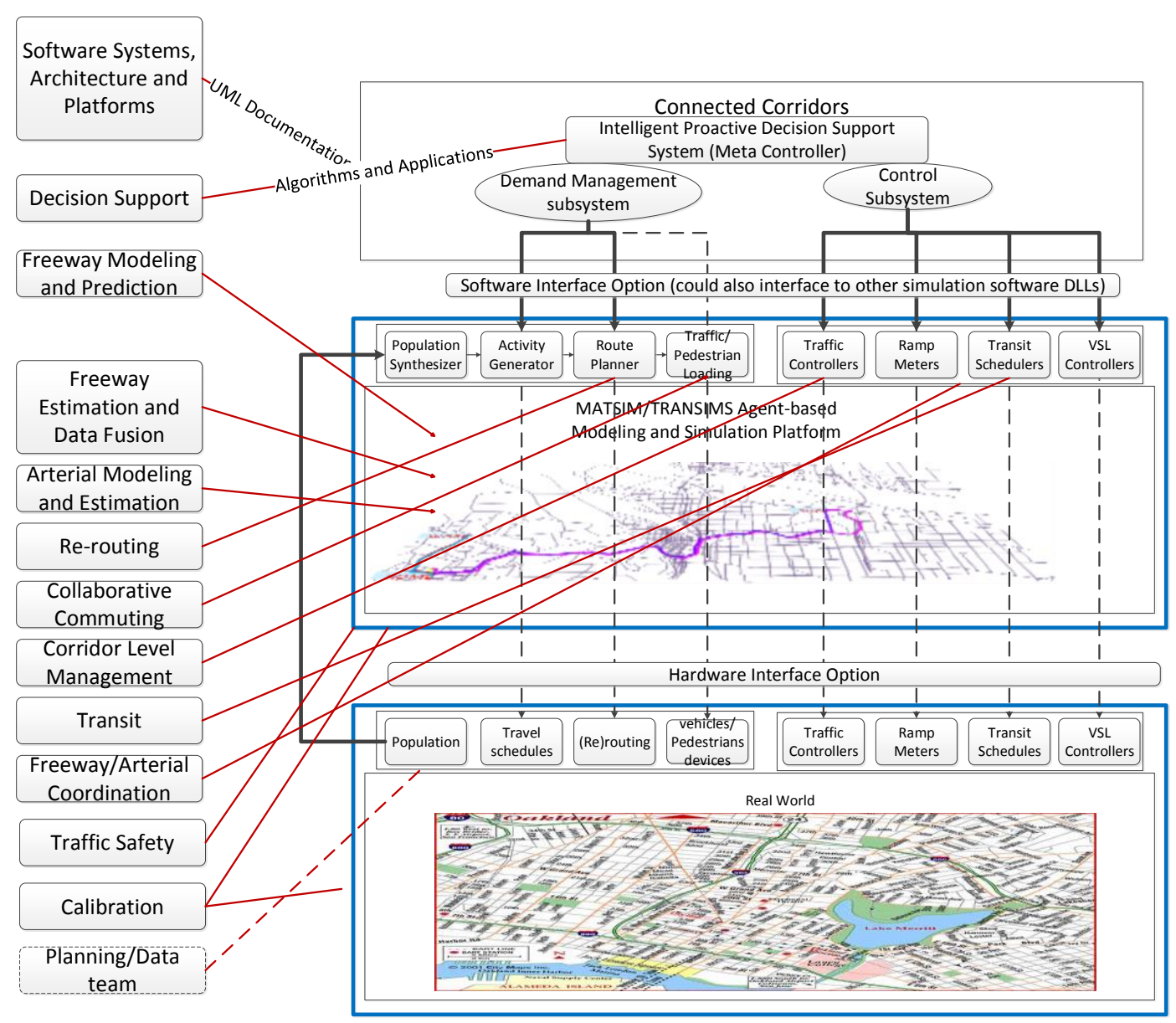

Figure 2. Overall Vision of Work

\subsection{Multi-Agent Transport Simulation (MATSIM)}

MATSim (Multi-Agent Transport Simulation) is a large-scale agent-based transport simulation framework developing jointly at TU Berlin, ETH Zurich, and Senozon Company. MATSim has been used in several studies and projects around the world (for examples see [5-10]). This open source simulation software is capable of simulating millions of agents or huge, detailed networks [11]. In the multi-agent simulation, each agent represented a round trip. Every model agent is assigned at least one plan: a series of activities and connecting trips. A plan is made step by step by behavioral modules inserting activity patterns, locations, mode choice decision, precise routes and times. Plans are processed into the traveler's minds as the "mental layer" of multi-agent simulation. Once all plans are completed, they are submitted to the traffic flow simulation to try the optimal assignment under the physical constraints caused by other agents. This is called the physical process as a "physical layer" of multi-agent simulation. During the traffic flow simulation, the performance of every agent is recorded. The utility value also known as the score of every plan is computed. Through mental and physical iterations, every agent attempt to modify its plan to get a larger score.

MATSim capabilities and functionalities have been noted in many studies. As some cases in point, Balmer et al., [12] simulated Switzerland network model containing about 7.1 million trips and 60000 links. It was a part of the MATSim research project. They showed detailed results of the simulation on the single agent level is obtained in 36 hours computation time [12]. Similarly, MATSim was applied by Meister et al., [13] in a largescale simulation of Switzerland network considering six million agents and over one million links. A realistic base case scenario, taking four modes of transport into account, 
was simulated, and results were validated with average workday count data [13]. Joubert et al., [6] used MATSim to simulate both commercial activity chains and private vehicles in Gauteng, South Africa. Comparison between the simulated model and observed vehicles chain from Global Positioning System were conducted, which revealed the geographical and temporal accuracy of the simulated model in MATSim. In another study, focusing on location choice module for shopping and leisure activities in MATSim, Horni et al., [12] validated the simulation results using a $10 \%$ sample of the Swiss network. They also indicated that MATSim has the potential to be used as a hypothesis testing tool as well as a planning tool [12]. Nagel and Flötteröd [14] focused on the evolution of traffic modeling from trips to behavioral travelers. They addressed some of the issues of most dynamic traffic assignment (DTA) models regarding behavior-based trips. The design of the MATSim simulation system was used to present how these issues can be addressed [14].

MATSim capabilities have been also compared to other traffic software. In this regard, Gao et al., [15] compared agent-based micro-simulation modeling and conventional fourstep modeling technique using MATSim and EMME/2 as the representatives of these approaches. They applied Greater Toronto and Hamilton Area network and actual demand data to investigate four indicators of the road network, namely travel time, travel distance, link volume, and link speed. The result of their study revealed that MATSim results are comparable to EMME/2. Regarding temporal point of view, MATSim was reported to be more realistic.

MATSim has the potential to be linked to other toolkits and modeling frameworks providing a substantial application ground. The following studies served as examples for utilization of MATSim integration capability. Hatzopoulou, Hao et al., [5] used MATSim for travel demand and traffic flow in conjunction with an activity-based travel demand model, TASHA (Travel Activity Scheduler for Household Agents), and vehicle emissions (MOBILE6.2C) and atmospheric dispersion (CALMET/CALPUFF) models. They evaluated vehicle emissions and greenhouse gases in the Greater Toronto Area (GTA) considering policy interventions and year of 2031 scenarios. In another effort, Waraich et al., [16] focused on charging of Plug-in Hybrid Electric Vehicles (PHEVs) applying micro simulation modeling of detailed traffic and electricity demand. They integrated MATSim and PMPSS to investigate three different charging strategies. The result of their effort is applicable to investigate capability of existing electric grids in response to increased future electricity demand by PHEVs [16]. Another example is the integration of Tel Aviv activity based model and MATSim agent-based dynamic framework by Bekhor et al., [17]. They utilized the best characteristics of both models; meaning disaggregate demand model of Tel Aviv and disaggregate supply representation of MATSim. They concluded that for the medium size network examined in their study, the dynamic assignment in MATSim is quite fast. Comparison at the aggregate level revealed that general planning and detailed case studies could both benefit from a more detailed behavioral modeling [17]. As the last example of studies regarding MATSim integration, Rieser [18] linked a detailed simulation of schedule-based transit into MATSim to overcome the limitation of MATSim in only modeling private cars. Computational issue, required changes, and extensions are detailed as well as providing an application of the integrated model [18].

Some studies have focused on improving the performance of the models in MATSim. For instance, Waraich et al., [19] introduced improvement and acceleration approaches to MATSim performance. The Presented methods were applied on Switzerland network including up to one million roads and 7.3 million agents [19]. In another study, presenting structural design and functionalities of MATSim in details, Balmer [20] implemented a preliminary implementation on a real world scenario placed in the greater Zurich area. He enhanced the design and implementation of the system focusing on structural issues, functional issues, and initial condition issues [20]. 


\subsection{Other agent-based Modeling Efforts in Transportation}

Other software and tools were also used to implement ABMS techniques in transportation research. Doniec et al., [21] proposed a multi-agent behavioral model in intersections according to the opportunistic individual behavior including norm violation and anticipatory individual abilities of simulated drivers that allow critical situations to be detected. The model was validated in several traffic conditions. Traffic in a real intersection was simulated and compared with the real flow in evaluation. In this highresolution modeling effort, the authors proposed that agents should coordinate their actions in order to avoid collisions in intersections. In the real world, Highway Code may not always be respected. Drivers move their cars opportunistically depending on the context and traffic situation ignoring the rules and satisfying their own desires temporarily. An agent searches for all vehicles with which are potentially in conflict and assesses its priority relationship with each of those vehicles. Speed differences between agents, impatience levels, vehicle positions, and traffic density are conditions for priority rules. Where the agent is concerned, agent speed, distance to collision point and evaluation on other conflict vehicles are factors to determine whether to make a violation or not.

Experiments were carried out to evaluate this method and were compared to Champion's [22]coordination mechanism as well. From the result, the behavioral model was reported to greatly improve the traffic simulation. The behavior model reduces oscillations; agents hesitate between two actions and alternate between one and the other, in this case, to cross the intersection or not, in terms of vehicle speed which means behavior mode works well according to its priority rules. In another experiment, the number of gridlock situations remained near zero in the majority of the time for simulated high density flows, which is logical in the real world.

Dia [23] proposed an agent-based approach to model individual driver behavior under the influence of real-time traffic information. Under the advanced traveler information systems (ATIS), the traveler gets responses to real time information about traffic conditions, accident delays, road work and route guidance. The agent behavior parameters including individual driver characteristics, knowledge, and preferences were identified through the responses of a survey implemented from a congested corridor in Brisbane, Australia. A simple agent-based route choice decision model within a microscopic traffic simulation tool was used to develop more complex behavior based on desire, intention, and individual difference of an agent under the real-time information. The objective of a driver was assumed to travel between an origin and destination with a given amount of information. Perception of information is in accordance with driver's preference, such as previously acquired knowledge and experience. Repetition of a choice depends on past and future satisfactory outcome or the feedback of anticipated outcomes. The paper uses real data including the complete set of drivers' choices to find out the characteristic of dynamic driver behavior from a driver behavior survey.

Hackney [24] proposed that social reasons including interactions between drivers can affect travel behavior. In order to construct hypothetical geographic interaction mode, agent simulation based on a MATSIM Toolbox (MatSim-T) was introduced to serve as a laboratory for scenario and hypothesis testing. A micro simulation on travelling agent factors was used to postulate different social interactions and observe feedbacks on short term travel decisions. The paper built social networks, summarized the model, and presented detailed validation results on the description of individual effects of socializing assumptions on travel behavior. A MATSIM model was constructed with a geographic world, transportation Network, a set of facilities, and a set of agents with plans consisting of each agent's activities and locations. The maximum utility for each agent's daily plan was realized by an evolutionary algorithm which favors successful outcomes of the agents' adaptation strategies. Agents got feedback from information, interactions and replanning. MATSIM focused on single daily travel. The iterative utility maximization 
should be viewed as an optimization algorithm seeking system relaxation given a certain utility function. The authors reported the results of several scenarios, with each scenario requiring tens of hours to run.

\subsection{Agent-Based Learning}

Nakayama [25] provided a route choice behavior of drivers in traffic assignments depending on a driver's memory content and rules . Agents simulate driver learning behavior based on their experiences and information provided. The behavior of agents and mechanism of transportation system were examined through experiments. An agent in this study cannot predict traffic state exactly on the trip, but an agent can learn how to choose a route according to its previous experience and preference. Nonlinear interaction between agents and system dynamics can reach a steady state through this learning process. In this paper, an if-then rule system was adopted as an agent decision-making. The if-then rule system was updated based on travel results through the agent learning process and the agent chose a route from the if-then rule system. The paper assumed that an agent's daily travel is from a fixed region to a destination and the only decision element is route choice. The model consists of an agent sub-model which simulates each agent's route choice with learning and a traffic state sub-model which determines traffic state based on agents' route choices and evaluates travel time.

An example of agent learning in MATSIM was conducted by Rieser [26], who proposed an agent-based method to get traveler information from writing out all agents plans instead of an OD matrix. MATSim was used to take the plans as input and iterates between the traffic flow simulation and the behavioral modules (route finding and time adjustment). The paper also compared the output (average volume capacity ration) with observed volume in Berlin and provided explanations for the errors. In this paper, individuals' data from an existing Activity Based Demand Generation (ABDG), based on Kutter-Model, was used to produce agent's plans for the region of Berlin. MATsim iterated between traffic flow simulation and the behavioral modules. The traffic flow simulation moved agents through the network according to the plans and generates events. Travel times, travel speeds, link densities, and other information could be calculated from these events. Each plan is evaluated by score according to how successful the agent performed. A percentage of agents were allowed to re-plan their daily plan with behavioral rules. Departure times and activity goals were modified to optimize individuals plan scores using time adjustment in the re-plans. Activity re-sequencing would change the order of the activities. The simulation stopped when agents' average score did not significantly improve.

\section{Modeling the Bay Area in MATSIM}

Figure 3 shows the general modeling framework in MATSIM. Both demand and supply information is used to produce the base case scenario. The simulation platform itself can be configured with different modeling options for the mobility model, scoring model, and the learning model used by agents. Since MATSIM is open source software, more in-depth modifications can be done through programming. In this preliminary effort, the base case scenario was produced to test the merits of using MATSIM in this project.

The network file contains a description of the network links and nodes. Nodes are defined by their coordinates, while links are defined by the link associated nodes. Link attributes include length, capacity (vehicles per hour), free-flow speed, and the number of lanes. This information was obtained from open street maps. The information could also be obtained from a commercial network map provider if higher accuracy is desired. The facilities in MATSIM define the location and opening hours for commercial destinations, such as shopping and leisure locations and should be obtained from business census. In our analysis, we used the TAZ-based origin-destination matrices to determine facility 
locations. A common set of opening hours was selected based on common practice in the area (e.g., malls closing at 9:00 p.m., leisure activities possible during the whole day).

The population data is used in MATSIM to establish the number of agents in the network, as well as the socioeconomic data of each agent. This data is typically obtained from the census data, where the house location of each agent could be obtained within reasonable accuracy. In our analysis, agents' house and work locations were obtained at the Transportation Analysis Zone (TAZ) level for about 2.1 million agents in the bay area. A synthesized location was then assigned to each agent within the 1454 TAZs in the Bay area. Data from the activity based model of the Metropolitan Transportation Commission (MTC) in San Francisco bay area were analyzed to synthesize a sample of initial activity plans for the agents. MATSIM tools were then used to map the travel activity plans to produce a synthesized set of travel plans for all simulated agents. Each agent initial plan included a set of travel activities, the origin and destination locations of each activity, purpose of each activity (e.g., work, leisure, shop, etc.), and activity durations. Each plan starts at home, and ends at home at the end of the day. Location of leisure and shopping activities, along with the open facility hours, were obtained from the MATSIM facilities information. MATSIM automatically references all activities with their nearest link to load the resulting demand over the network. MATSIM iteration results in different assignment of agents to routes and travel times while striving to meet the required schedule. This is an additional advantage of using ABMS over microscopic simulation models, or dynamic traffic assignment, where only routes are changed. In addition, the ABMS approach accounts for drivers learning during inter- or intra-day driving.

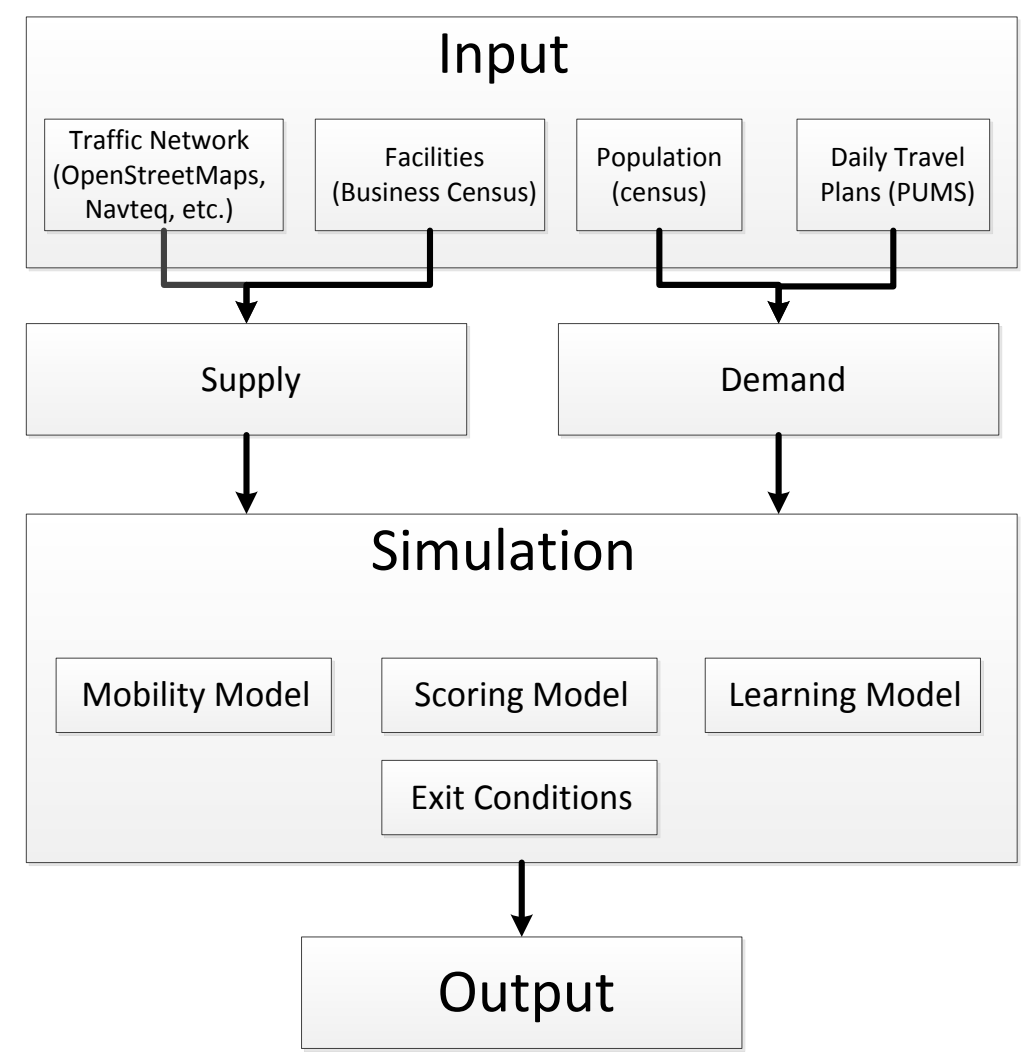

Figure 3. MATSIM Modeling Steps 


\subsection{Simulated Network}

Figure 4 shows the simulated network of the San Francisco bay area. Different modeling resolutions were used to model the dense areas of the network as shown in the figure. The modeled network consisted of 31,608 nodes and 73,711 links.

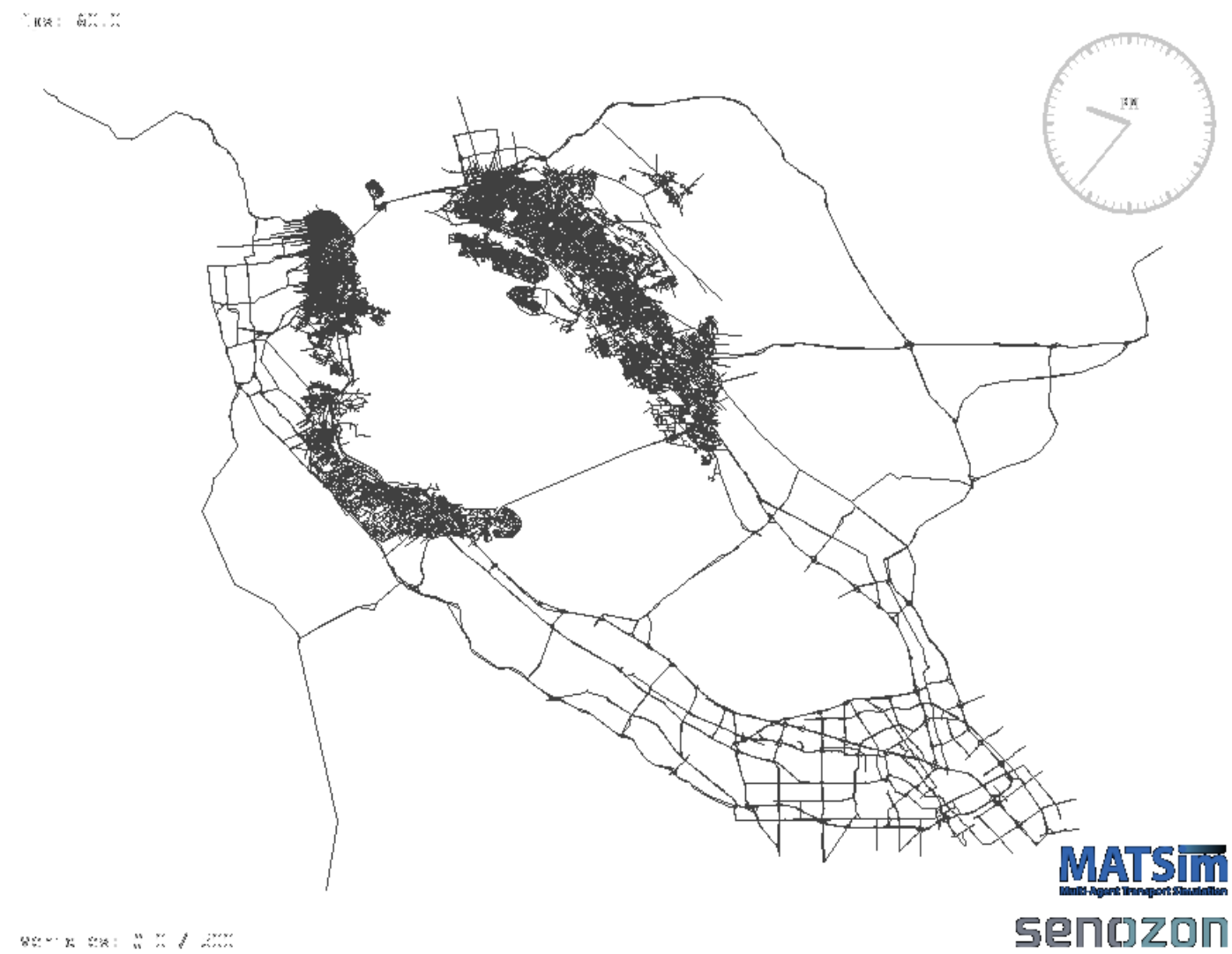

Figure 4. MATSIM Network of the Bay Area

\subsection{Results and Assessment}

The bay area city road network was simulated to assess potential merits of the model. The average score of all agent plans shown in Figure 5 provide a good overview of the iterations' progress. MATSIM uses the scores in each iteration as a feedback mechanism to the agents who might in turn change or produce new travel plans. This iteration process results in agents "learning" from their experience to shift their travel behavior. Options available to each agent to choose from could be configured to include different demand strategies. The figure shows that agents are learning in the simulation, and is an indication that detailed runs with more interactions and different demand management strategies could provide more insight into the system's behavior. This in turn could be utilized to produce and test different demand strategies. 


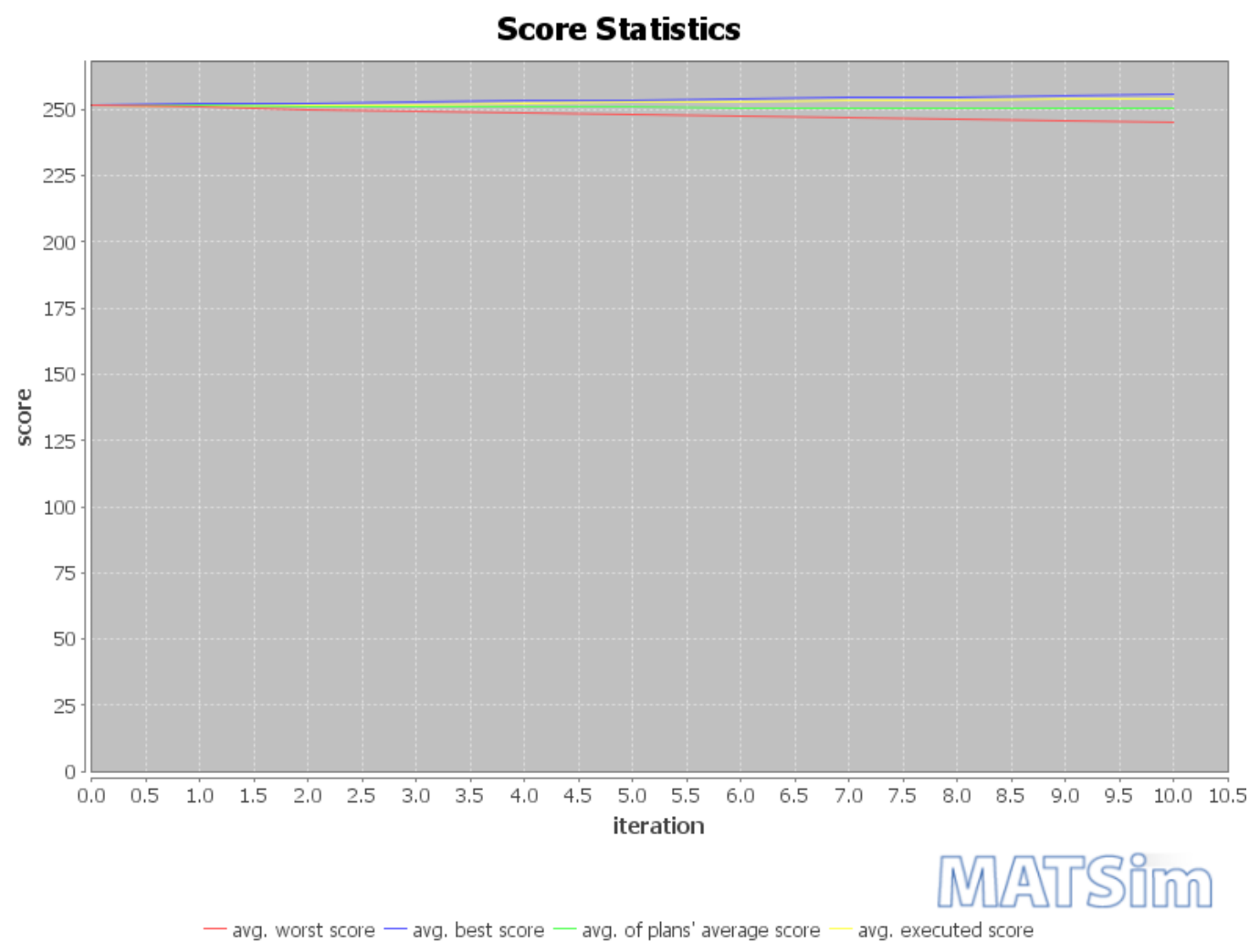

Figure 5. MATSIM Scoring Statistics

Figure 6 shows a summary of the volume variation in the MATSIM network. The figure shows the variation of demand by time, and corresponds to the anticipated peaks in traffic volume that can be observed in a network. The ability of the software to produce this sort of figures serves multiple purposes: (1) calibration of the traffic models used can be conducted using actual traffic counts where and when available, (2) the effect of different demand management strategies can be visualized (and analyzed) both for the network and for specific links, and (3) the impact of different control strategies on the overall system performance can be analyzed. These capabilities can then be leveraged to produce intelligent meta-controllers for the whole connected corridor. 


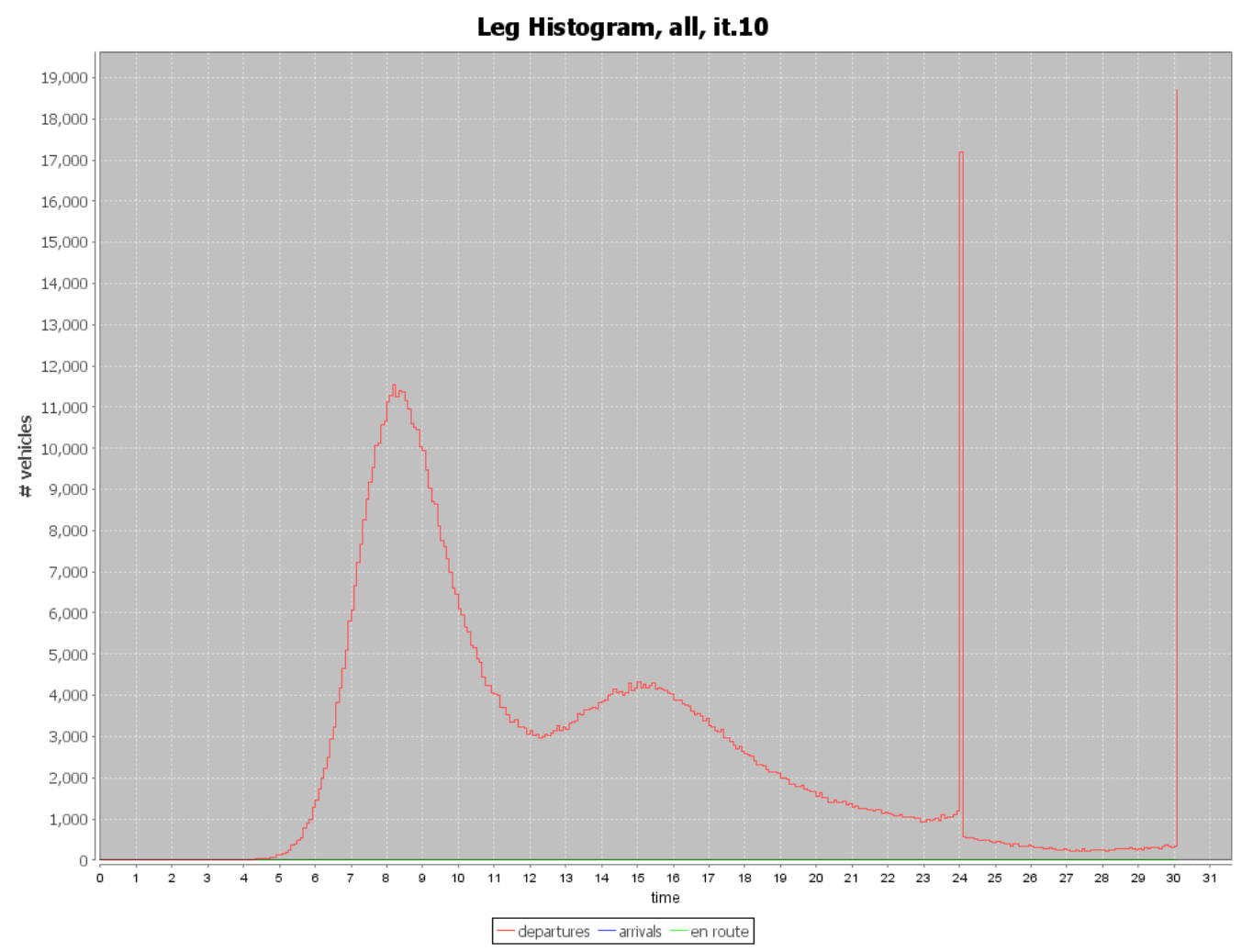

Figure 6. MATSIM Volume Variation

\section{Conclusion and Future Steps}

This paper provided a summary of a research effort to investigate the merits of using agent-based modeling and simulation techniques in a connected corridor. Potential use and integration of ABMS tools with research already in progress was described. The base case model of the bay area and used data was shown. The results show potential advantages for using MATSIM as a supporting tool for the research.

Possible future steps related to this effort include:

-Verification and calibration of the network using actual traffic counts and travel survey data,

- Use of different route choice and dynamic traffic assignment settings in the model, based on state-of-the-art models developed by the team,

-Evaluation of the mobility model used in MATSIM, and potentially improving the tool,

- Test and evaluate the combination of demand and supply strategies for different simulation scenarios.

\section{Acknowledgments}

This work was performed in collaboration with PATH at UC-Berkeley in Fall 2012 as part of a faculty research leave assignment. 


\section{References}

[1] E. Bonabeau, "Agent-based modeling: Methods and techniques for simulating human systems", vol. 99, (2002).

[2] J. Holland, "The Global Economy as an Adaptive Process", The Economy As a an Evolving Complex System, (1988).

[3] A. I. a. A. C. A. Committee, "Artifical Intelligence in Transportation", Artifical Intellegence, T.R. Board, Editor, Washington, DC., (2007), pp. 88.

[4] A. I. a. A. C. A. Committee, "Artifical Intelligence in Transportation", Artifical Intellegence, T.R. Board, Editor, Washington, DC., (2012), pp. 58-64.

[5] M. Hatzopoulou, J. Hao and E. Miller, "Simulating the impacts of household travel on greenhouse gas emissions, urban air quality, and population exposure", Transportation, vol. 38, no. 6, (2011), pp. 871887.

[6] J. Joubert, P. Fourie and K. Axhausen, "Large-Scale Agent-Based Combined Traffic Simulation of Private Cars and Commercial Vehicles", Transportation Research Record: Journal of the Transportation Research Board, 2168(-1), (2010), pp. 24-32.

[7] D. Grether, S. Fürbas and K. Nagel, "Agent-based Modelling and Simulation of Air Transport Passenger Demand", Submitted to ABMTRANS 2013, (2013).

[8] P. J. Fourie and J. W. Joubert, "The first agent steps in agent-based transport planning", In the 28th Southern African Transport Conference, South Africa, (2009).

[9] M. Rieser, K. Nagel, U. Beuck, M. Balmer and J. Rümenapp, "Agent-Oriented Coupling of ActivityBased Demand Generation with Multiagent Traffic Simulation", Transportation Research Record: Journal of the Transportation Research Board, 2021, (2007), pp. 10-17.

[10] M. Balmer, K. Axhausen and K. Nagel, "Agent-Based Demand-Modeling Framework for Large-Scale Microsimulations", Transportation Research Record: Journal of the Transportation Research Board, 1985, (2006), pp. 125-134.

[11] K. Nagel. MATSim (Multi-Agent Transport Simulation). 2012; Available from: http://www.matsim.org/.

[12] A. Horni, D. M. Scott, M. Balmer and K. W. Axhausen, "Location Choice Modeling for Shopping and Leisure Activities With MATSim: Combining Micro-simulation and Time Geography", Transportation Research Record: Journal of the Transportation Research Board, pp. 87-95, (2009).

[13] K. Meister, M. Balmer, F. Ciari, A. Horni, M. Rieser, R. A. Waraich and K. W. Axhausen, "Large-scale agent-based travel demand optimization applied to Switzerland", including mode choice in 12th World Conference on Transportation Research, Lisbon, (2010).

[14] K. Nagel and G. Flötteröd, "Agent-based traffic assignment", going from trips to behavioral travelers in 12th International Conference on Travel Behaviour Research (IATBR), Jaipur, (2009).

[15] W. Gao, M. Balmer and E. Miller, "Comparison of MATSim and EMME/2 on Greater Toronto and Hamilton Area Network, Canada", Transportation Research Record: Journal of the Transportation Research Board, 2197, (2010), pp. 118-128.

[16] R. A. Waraich, M. D. Galus, C. Dobler, M. Balmer, G. Andersson and K. W. Axhausen, "Plug-in hybrid electric vehicles and smart grids: Investigations based on a microsimulation", Transportation Research Part C: Emerging Technologies, 28(0), (2013), pp. 74-86.

[17] S. Bekhor, C. Dobler and K. W. Axhausen, "Integration of Activity-Based with Agent-Based Models", an Example from the Tel Aviv Model and MATSim in 90th Annual Meeting of the Transportation Research Board, Washington, D.C., (2011).

[18] M. Rieser, "Adding Transit to an Agent-Based Transportation Simulation: Concepts and Implementation", VSP, TU Berlin, Germany, (2010).

[19] R. A. Waraich, D. Charypar, M. Balmer and K. W. Axhausen, "Performance improvements for large scale traffic simulation in MATSim", in 9th Swiss Transport Research Conference, Monte Verita, (2009).

[20] M. Balmer, "Travel demand modeling for multi-agent transport simulations: algorithms and systems", Computer Science, ETH Zurich, Switzerland, (2007).

[21] A. Doniec, R. Mandiau, S. Piechowiak and S. Espie, "A behavioral multi-agent model for road traffic simulation", Engineering Applications of Artificial Intelligence, vol. 21, no. 8, (2008), pp. 1443-1454.

[22] A. Champion, M.-Y. Zhang, J. M. Auberlet and S. Espie, "Behavioral simulation: Towards high-density network traffic studies", 3rd International Conference on Traffic and Transportation Studies, ICTTS, Guilin, China: American Society of Civil Engineers, (2002) July 23-25.

[23] H. Dia, "An agent-based approach to modelling driver route choice behaviour under the influence of real-time information", Transportation Research Part C: Emerging Technologies, vol. 10, no. 5-6, (2002), pp. 331-349.

[24] Highway Capacity Manual, National Research Concil, TRB, (2000).

[25] S. Nakayama, "An Analysis of Transportation System mechanism using the Agent-Based Simulation", pp. 9, (2008). 
[26] M. Rieser, K. Nagel, U. Beuck, M. Balmer and J. Rumenapp, "Agent-oriented coupling of activity-based demand generation with multiagent traffic simulation", Transportation Research Record, (2021), (2007), pp. 10-17.

\section{Authors}

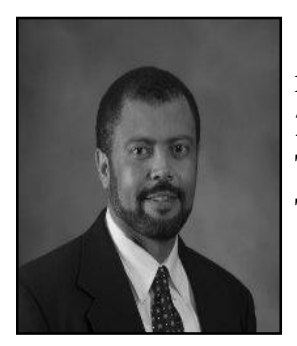

Montasir Abbas is an Associate Professor at Virginia Tech. He received his Ph.D. in Civil Engineering from Purdue University in 2001. He has previously worked as an Assistant Research Engineer at Texas Transportation Institute and as a Visiting Assistant Professor at Texas A\&M. His e-mail is <abbas@vt.edu>.

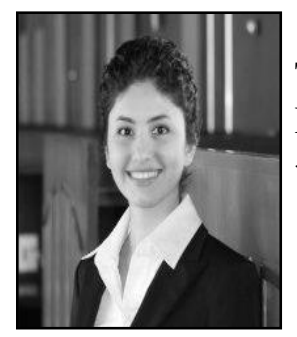

Sahar Ghanipoor Machiani is a Research Associate at Virginia Tech Transportation Institute. She received her Ph.D. in Civil Engineering from Virginia Tech in December 2014. Her e-mail is <sahargh@vt.edu>. 
International Journal of Transportation

Vol.4, No.1 (2016) 